\title{
A Novel de novo FZD2 Mutation in a Patient with Autosomal Dominant Omodysplasia
}

\author{
Seval Türkmen ${ }^{a, b}$ Malte Spielmann ${ }^{a, c}$ Nilay Güneş $^{e} \quad$ Alexej Knaus $^{a, c, d}$ \\ Ricarda Flöttmann ${ }^{a}$ Stefan Mundlos ${ }^{a, c}$ Beyhan Tüysüz \\ ${ }^{a}$ Institut für Medizinische Genetik, Charité Universitätsmedizin Berlin, ${ }^{b}$ Labor Berlin Charité Vivantes Berlin, \\ 'Max Planck Institute for Molecular Genetics, and dBerlin-Brandenburg School for Regenerative Therapies (BSRT),

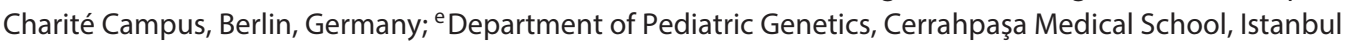 \\ University, Istanbul, Turkey
}

\section{Established Facts}

- Autosomal dominant omodysplasia (OMOD2) is a rare skeletal dysplasia, characterized by facial dysmorphism and shortness of the upper extremities and first metacarpals.

- Recently, a nonsense mutation in the FZD2 gene has been described only in one family with OMOD2, and the authors claimed that FZD2 is responsible for this disease.

\section{Novel Insights}

- This is the second report which supports the findings of the first family and points out that heterozygous FZD2 mutations may be disease-causing for OMOD2.

\section{Keywords}

Frizzled-2 · FZD2 · OMOD2 · Omodysplasia

\begin{abstract}
We described a heterozygous de novo mutation (G434V) in the frizzled class receptor 2 (FZD2) gene in a patient with distinct facial features including hypertelorism, bilateral cleft lip/palate, short nose with a broad nasal bridge, microretrognathia, and bilateral shortness of the upper limbs, first
\end{abstract}

\section{KARGER}

(C) 2017 S. Karger AG, Basel

E-Mail karger@karger.com

www.karger.com/msy metacarpal bones, and middle phalanges of the 5th digits. The findings of our patient were compared to an autosomal dominant omodysplasia (OMOD2) family with FZD2 mutation reported in the literature. OMOD2 is a rare skeletal dysplasia and characterized by facial dysmorphism and shortness of the upper extremities and first metacarpal bones. This is the second report which supports the findings of the first family described and points out that heterozygous FZD2 mutations may be disease-causing for OMOD2.

(c) 2017 S. Karger AG, Basel

Beyhan Tüysüz

Çocuk Kliniği, Cerrahpaşa Tip Fakültesi

Istanbul Üniversitesi

Istanbul 34098 (Turkey)

E-Mail beyhan@istanbul.edu.tr 
Fig. 1. Photographs of the patient. a Hypertelorism, bilateral cleft lip and palate, broad nasal bridge, microretrognathia, short upper and lower limbs, and pes valgus of the right foot at neonatal period. b Note caféau-lait spots and freckling on the right side of the trunk, short first and fifth fingers, short arm, and toe anomalies at age 21 .
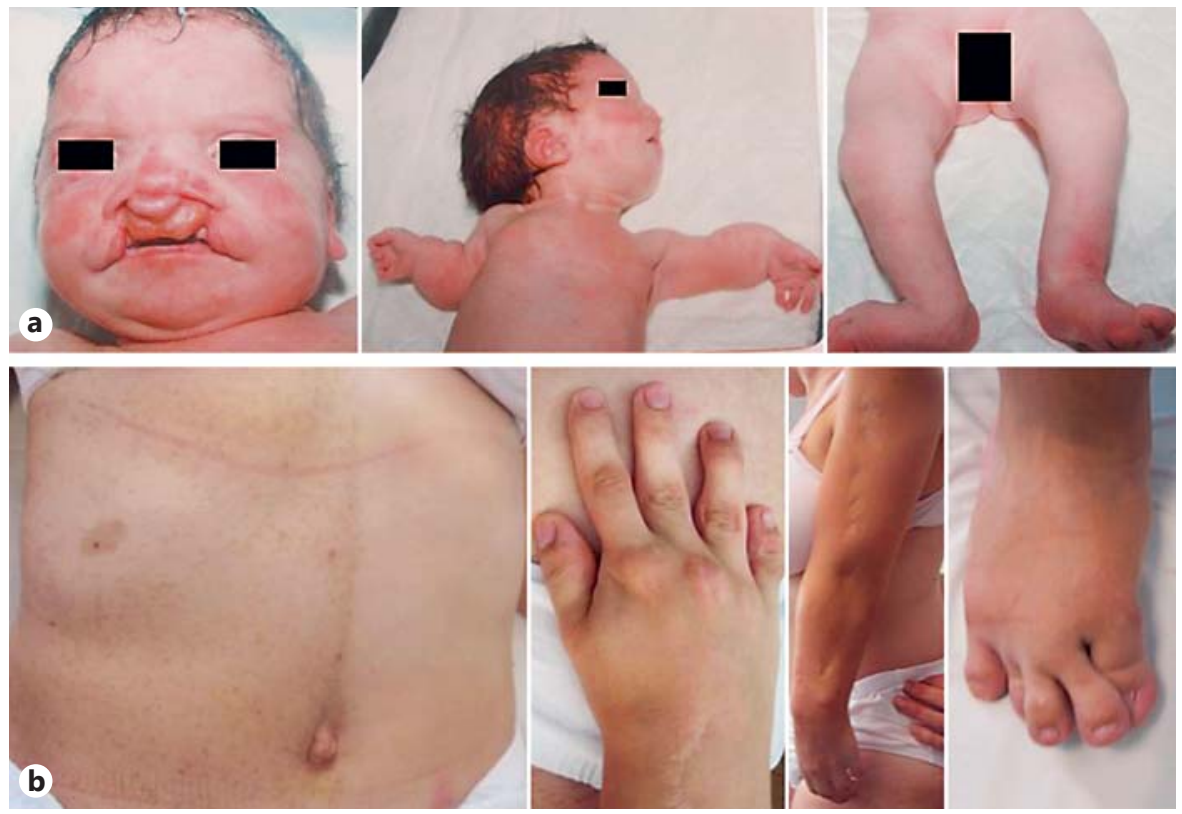

Omodysplasia (OMOD) is a rare skeletal dysplasia characterized by shortened limbs, especially the upper extremities, and craniofacial dysmorphism, including hypertelorism and a depressed nasal bridge with short nose and a long philtrum. Both autosomal recessive (OMOD1; OMIM 258315) and autosomal dominant inheritance (OMOD2; OMIM 164745) have been described, thus suggesting genetic heterogeneity [Maroteaux et al., 1989; al Gazali and Abou al-Asaad, 1995; Venditti et al., 2002; Elçioglu et al., 2004; Gordon et al., 2014]. Homozygous or compound heterozygous mutations in the heparan-sulfate proteoglycan glypican 6 gene (GPC6) were shown to cause OMOD1 [Campos-Xavier et al., 2009]. Recently, next-generation whole exome sequencing of a proband and her affected daughter with OMOD2 revealed a de novo heterozygous nonsense mutation (p.Trp548*) in the FZD2 gene. Further analysis of the mutation showed a clear decrease in canonical Wnt signaling as a result of the mutation [Saal et al., 2015]. This gene codes the Frizzled-2 protein which acts as a Wnt receptor and is expressed during facial and skeletal development in multiple model systems. Although the heterozygous mutation in the FZD2 gene is identified in one family as a novel cause for OMOD2 [Saal et al., 2015], this condition has been included in the group of mesomelic and rhizo-mesomelic dysplasias in the last nosology and classification of skeletal genetic disorders [Bonafe et al., 2015].

Autosomal Dominant Omodysplasia
Here, we present the clinical and molecular genetic data of a Turkish patient with OMOD2 in whom whole exome sequencing revealed a novel mutation in the FZD2 gene.

\section{Clinical Report}

The newborn girl was consulted due to short limbs and a cleft lip and palate. She is the first child of unrelated healthy parents aged 34 and 26. She was born at term, and her birth length and weight were $52 \mathrm{~cm}$ and $3,200 \mathrm{~g}$, respectively. There was no family history of cleft lip/palate, short limbs, café-au-lait spots, or neurofibromas. Physical examination showed dysmorphic facial features including a large forehead, round face, hypertelorism, low-set ears, bilateral cleft lip and palate, microretrognathia, rhizomelic and mesomelic shortening of the upper extremities, bilateral ulnar deviation, mild shortening of lower extremities, and pes valgus of the right foot (Fig. 1a). She had normal external genitalia. There was no abnormality on her cranial and abdominal ultrasonography as well as echocardiography. Chromosome analysis showed a normal female karyotype.

While language and mental development were appropriate for age, motor development was delayed on her follow-up. She underwent recurrent surgical repair of cleft lip and palate as well as extension operations on the upper limbs until 12 years of age.

Around 6 years of age, a spinal MRI was performed due to ataxia, and a lesion was detected at the level of C6 and C7. The lesion was diagnosed as neurofibroma after pathological examination. At the age of 14, a nontender and pigmented lesion was also detected on the lumbar region of skin. This lesion was diagnosed as a neurofibroma as well. 

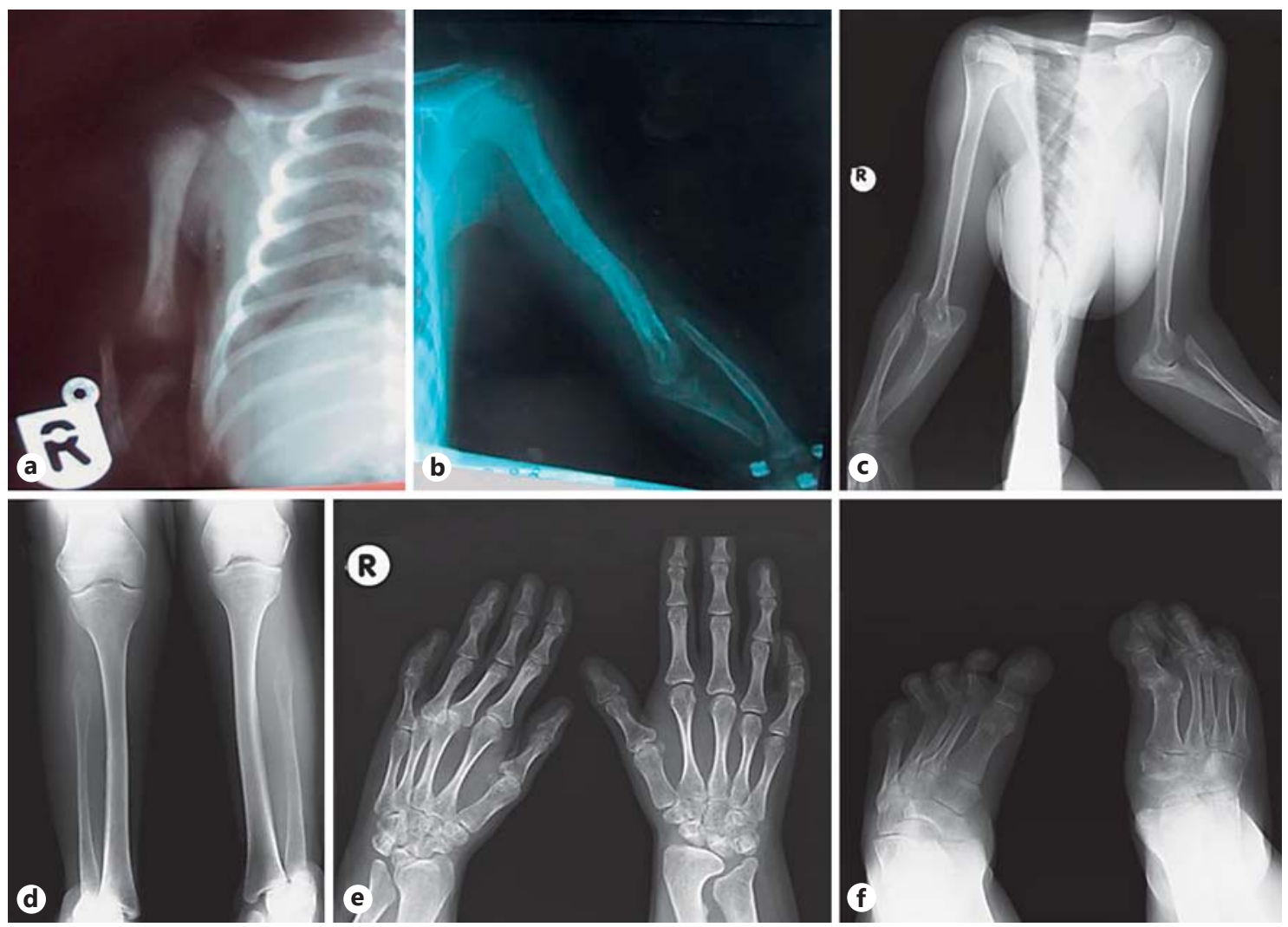

Fig. 2. Skeletal radiographs showing short humerus and ulna and radial dislocation of the patient. a Neonatal period. b 5 years old. c 21 years old. Note the short fibula with hypoplastic proximal parts (d), short first metacarpal bone of the left hand, short second phalanges of the fifth fingers bilaterally, and poorly formed radial and ulnar metaphysis (e), short first metatarsal bone, and dislocation of the first proximal phalangeal joint of the right foot and metatarsus adductus bilaterally (f) at the age of 21 .

At the last examination, her stature was $145 \mathrm{~cm}(-3 \mathrm{SD})$ at age 21 . She had normal intelligence. She started having menstrual cycles at 14 years of age, and her cycles were regular. Physical examination showed a prominent forehead, hypertelorism, low-set ears, broad nasal tip, operated cleft lip and palate, short upper limbs, bilateral short thumbs and 5th fingers, ulnar deviation, limited forearm pronation and supination, diffuse freckling of the skin and 4 café-au-lait spots larger than $15 \mathrm{~mm}$ only on the right side of the trunk. She also had bilateral metatarsus adductus and a short first toe which is overlapped by the second toe on the right foot (Fig. 1b). Her eye and hearing examinations were normal.

\section{Radiological Studies}

The radiography of the upper extremities revealed short humerus with narrowing diaphysis towards distal metaphysis, proximal radial dislocation, short radius, and very short ulna at neonatal period (Fig. 2a) and at 5 years of age (Fig. 2b). Narrowing iliac wings and mild subluxation of femoral heads as well as mild coxa vara were observed in pelvic X-rays at the age of 1 year.

Skeletal surveys at her last examination showed bilateral radial dislocation, short radius and very short ulna (Fig. 2c), short first metacarpal bones and bilateral short middle phalanges of the 5 th fingers, poorly formed radial and ulnar metaphyses (Fig. 2d), short fibula with proximal hypoplasia (Fig. 2e), short first metatarsal bones, and dislocation of the first proximal phalangeal joint of the right foot and bilateral metatarsus adductus (Fig. 2f). Vertebral and pelvic $\mathrm{X}$-rays were normal.

\section{Materials and Methods}

\section{Next-Generation Sequencing Analysis}

Since no clinical diagnosis was established, genomic DNA was isolated from peripheral blood samples of the patient and, following Agilent SureSelect All Exon kit V4 enrichment, was subjected to next-generation sequencing on Illumina's HiSeq (paired-end 150 bp). Subsequently, a computational method termed Phenotypic Interpretation of eXomes (PhenIX), which evaluates variants based on population frequency and predicted pathogenicity and then ranks the genes according to variant score and a clinical relevance score, was used to prioritize candidate genes [Zemojtel et al., 2014]. To calculate the clinical relevance score, we entered the following human phenotype ontology terms HP0005792 (humeral hypoplasia, short upper arms, humeral shortening, hypoplastic humerus, and short humeri) [Robinson et al., 2008]. 
Fig. 3. In silico modeling of the $\mathrm{G} 434 \mathrm{~V}$ mutation in FDZ2. In the wild type, the Gly434 is represented as red spheres in the TM5 helix (orange). The sterically least problematic configuration shows steric hindrances of Val434 with the neighboring helix (yellow).

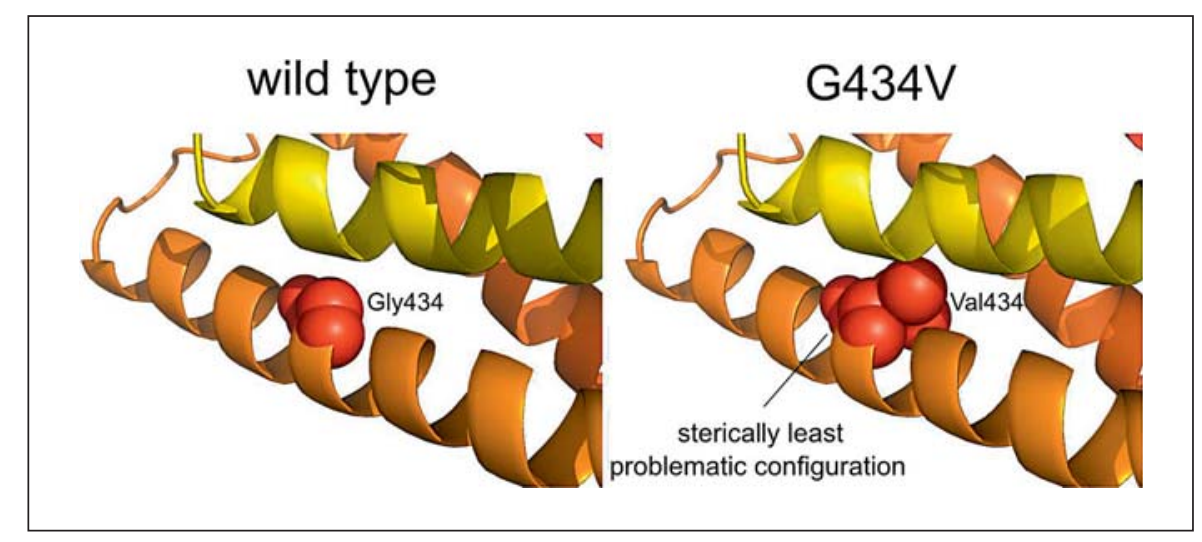

Sanger Sequencing Analysis

Genomic DNA from the patient and his parents was extracted from peripheral blood leukocytes using standard protocols. The coding region and flanking intronic sequences of exon 1 of the FZD2 gene were amplified and sequenced using standard protocols. Primers and PCR conditions are available upon request. The sequencing results were processed with the 4 Peaks software.

\section{Mutation Modeling}

The model of FZD2 was generated by I-TASSER and RaptorX using the NMR structure of the smoothened receptor (SMO; class frizzled (class F) G-protein-coupled receptor; PDB-ID 4JKV) [Zhang, 2008; Roy et al., 2010; Källberg et al., 2012; Yang and Zhang, 2015; Yang et al., 2015]. The sequence homology between SMO and FZD2 is 23\% identical overall and 60\% in the transmembrane helix 5. The glycine $(G)$ residue is highly conserved in SMOs in the FLxxG motif in the transmembrane helix 5. The glycine to valine mutation was modeled with PyMol v1.3.

\section{Results}

We identified a heterozygous missense mutation (c.1301G>T) in exon 1 of the FZD2 gene converting glycine at position 434 to valine $(\mathrm{G} 434 \mathrm{~V})$. The mutation was de novo and was not present in the parents. The variant is predicted to cause a p.Gly $434 \mathrm{Val}$ alteration to the protein sequence at a highly conserved position (Fig. 3) and is not present at any frequency in the NHLBI Exome Sequencing Project Exome Variant Server (EVS), $1000 \mathrm{Ge}-$ nomes (version 3), or 274 in-house exomes of mixed ancestry. This mutation was validated by Sanger sequencing. Bioinformatics programs (PolyPhen-2, SIFT, and MutationTaster) predicted the mutation to be deleterious.

The G434V substitution resides in the 5th transmembrane helix pointing inwards. Figure 3 shows a NMR-de- rived structure of the FZD2 gene. The in silico introduction of the G434V mutation, a relatively large hydrophobic amino acid, resulted in severe steric problems and shows the mutated protein configuration with the least steric hindrances.

There was no pathogenic variant after the reexamination of the exome data, focusing on both NF1 and NF2 genes.

\section{Discussion}

The bone morphogenetic protein (BMP), wingless-integrated (Wnt), sonic hedgehog (Shh), and fibroblast growth factor (FGF) pathways play a critical role in embryonic bone formation [Berendsen and Olsen, 2015]. Glypicans are proteoglycans located on the cell surface, where they regulate the activity of the signaling by ligands of the BMP, FGF, Shh, and Wnt pathways, through protein-protein and protein-carbohydrate interactions [Filmus and Capurro, 2014]. Wnt proteins constitute a large family of secreted ligands that control developmental processes in animals [Logan and Nusse, 2004]. Heterozygous mutations in the WNT5A gene cause autosomal dominant Robinow syndrome, characterized by mesomelic shortening, short stature, genital anomalies, and facial dysmorphism - including hypertelorism, upslanting palpebral fissures, and a short upturned nose with broad nasal bridge [Person et al., 2010]. Homozygous mutations in the GPC6 gene were shown to cause OMOD1 [Campos-Xavier et al., 2009]. Recently, Saal et al. [2015] demonstrated an autosomal dominant form of omodysplasia, which is caused by mutations in the FZD2 gene and is also important for the Wnt5a signaling. Wnt5a regulates multiple intracellular signaling pathways by binding to the

Mol Syndromol 2017;8:318-324 DOI: $10.1159 / 000479721$ 
Table 1. Comparison of the clinical and radiological findings of the present patient with those of reported patients

\begin{tabular}{|c|c|c|c|c|c|c|c|c|c|}
\hline \multirow[t]{2}{*}{ Features } & \multirow[t]{2}{*}{ Present patient } & \multicolumn{2}{|c|}{ Saal et al. (2015) } & \multirow{2}{*}{$\begin{array}{l}\text { Gordon et al. } \\
\text { (2014) }\end{array}$} & \multicolumn{2}{|c|}{ Venditti et al. (2002) } & \multicolumn{3}{|c|}{ Maroteaux et al. (1998) } \\
\hline & & patient 1 & patient 2 & & patient 1 & patient 2 & patient 1 & patient 2 & patient 3 \\
\hline Age of last examination & 21 years & 25 years & 6 years & 48 years & 25 years & 1 month & newborn & 27 years & 10 years \\
\hline Gender & $\mathrm{F}$ & $\mathrm{F}$ & M & $\mathrm{F}$ & $\mathrm{F}$ & M & M & $\mathrm{F}$ & $\mathrm{F}$ \\
\hline Height, percentile & $<5$ & 10 & $<5$ & $<5$ & 25 & 25 & 5 & 10 & 10 \\
\hline \multicolumn{10}{|l|}{ Facial features } \\
\hline Round face & + & + & + & - & + & NA & + & NA & + \\
\hline Prominent forehead & + & + & + & + & + & + & - & - & + \\
\hline Hypertelorism & + & - & - & - & + & + & + & + & - \\
\hline Broad nasal bridge & + & + & + & + & + & + & + & - & - \\
\hline \multicolumn{10}{|l|}{ Small nose with broad } \\
\hline nasal tip & - & + & + & + & + & + & + & + & + \\
\hline Long philtrum & - & + & + & + & + & + & - & - & - \\
\hline Microretrognathia & + & + & + & + & - & + & - & - & - \\
\hline Cleft lip/palate & + & + & - & - & - & - & - & - & - \\
\hline \multicolumn{10}{|l|}{ Skeletal features } \\
\hline Short humeri & + & + & + & + & + & + & + & + & + \\
\hline Radial dislocation & + & + & + & + & + & + & + & + & + \\
\hline Limited elbow joints & + & + & + & + & - & NA & + & + & + \\
\hline Short ulnae & + & NA & + & - & + & + & + & + & + \\
\hline \multicolumn{10}{|l|}{ Short first metacarpal } \\
\hline bones & + & NA & + & + & + & + & + & + & + \\
\hline Short fibulae & + & NA & + & - & - & - & - & - & - \\
\hline Genital anomalies & - & + & - & + & + & + & + & - & - \\
\hline FZD2 mutation & $\begin{array}{l}\text { c. } 1301 \mathrm{G}>\mathrm{T} \\
\text { (p.Gln } 434 \mathrm{Val})\end{array}$ & \multicolumn{2}{|c|}{$\begin{array}{l}\text { c. } 1644 \mathrm{G}>\mathrm{A} \\
\text { (p.Trp548*) }\end{array}$} & NA & NA & & NA & & \\
\hline
\end{tabular}

NA, not assessed. +, present; -, absent.

Frizzled-2 protein. The causal p.Trp548* mutation in the published family by Saal et al. [2015] is predicted to result in a truncation of the Frizzled-2 protein at the periphery of the canonical Dishevelled interaction domain, which may have significant consequences for canonical signaling. In canonical Wnt signaling, Frizzled binding to Dishevelled requires a conserved motif, KTxxxW, in the intracellular portion of the Frizzled receptor. The tryptophan residue $(\mathrm{W})$ in the KTxxxW motif is the site of the nonsense mutation in their patients. The G434V substitution identified in our patient is located in the highly conserved transmembrane helix 5 and probably severely impairs the correct folding and assembly of the neighboring transmembrane domains and thereby inhibits correct anchoring of FZD2 in the cell membrane [Tauriello et al., 2012].

OMOD1 associated with severe short stature and developmental delay differs clinically from the dominant form presenting with normal or mild short stature and normal intelligence. OMOD2 is a very rare condition and characterized by short upper extremities, hypoplastic distal humeri, dislocated radial head, limited elbow flexion/ extension, short first metacarpal bones, variable degrees of genital anomalies, and distinct facies including hypertelorism, a short nose with broad nasal bridge and tip, and microretrognathia. Only 8 patients from 4 families were diagnosed as OMOD2 until today [Maroteaux et al., 1989; Venditti et al., 2002; Gordon et al., 2014; Saal et al., 2015]. Venditti et al. [2002] emphasized that the facial and skeletal features of this rare disorder were similar to autosomal dominant Robinow syndrome, and the patients with OMOD2 may have been diagnosed previously as autosomal dominant Robinow syndrome.

We compared the clinical and radiological findings of our patient with those reported patients in Table 1. The patient presented here shares clinical features with the family carrying the FZD2 mutation (proband and her daughter) [Saal et al., 2015], such as a short humerus, radial dislocation, short ulna and first metacarpal bone, short fibula, and typical facial features including hypertelorism, a prominent forehead, short nose with a broad nasal bridge, and cleft lip and palate.

Unlike other reported patients who had no molecular studies, both our patient and patient 1 described by Saal et al. [2015] had bilateral cleft lip/palate as a common finding. They considered that this finding was not likely 
a part of the OMOD2 phenotype, and they had reexamined the exome data focusing on genes of the Wnt signals which were crucial for craniofacial development [GeethaLoganathan et al., 2009; Sisson and Topczewski, 2009; Yao et al., 2011]. Although they had found 7 variants in 5 WNT genes, none of these variants in the WNT genes were predicted to have an impact on the bioinformatics analysis. However, the presence of a cleft lip and palate in our patient suggests that this finding may be a symptom of OMOD2.

Although it has been reported that OMOD2 patients have rhizomelic shortness of the upper limbs, our patient and patients reported by Saal et al. [2015] also have rhizomesomelic shortening. The radiography of the upper extremities revealed short humerus with narrowing diaphysis towards distal metaphysis, proximal radial dislocation, and short radius and ulna. Short humerus with the abovementioned narrowing is a typical radiological finding in this disorder. Mild lower-limb involvement was reported in some cases. Our patient also had short fibula with hypoplastic proximal parts. Final statures were reported to be low-normal and normal in 4 patients [Maroteaux et al., 1989; Venditti et al., 2002; Gordon et al., 2014; Saal et al., 2015]. Our patient reached a final height of $145 \mathrm{~cm} \mathrm{(-3}$ $\mathrm{SD})$. A short first metacarpal bone is a typical finding in this syndrome. We observed a short first metacarpal bone and short middle phalanges of the 5 th fingers in our patient; the latter has been reported in a patient by Venditti et al. [2002].

Genital hypoplasia was reported in 5 out of 8 patients [Maroteaux et al., 1989; Venditti et al., 2002; Gordon et al., 2014; Saal et al., 2015]. Our patient had normal genitalia.

Although a cleft lip and palate as well as genital hypoplasia had been observed in patient 1 by Saal et al. [2015], the daughter, who had no cleft lip/palate and normal genitalia, had the same mutation in the FZD2 gene. These findings were considered as an intrafamilial variability in this disorder.

The patient presented here also developed a plexiform neurofibroma at the level of $\mathrm{C} 6 / 7$ in the medulla spinalis and segmental neurofibromatosis with diffuse freckling and 4 café-au-lait spots. The spots were larger than 15 $\mathrm{mm}$ and appeared only on the upper right side of the trunk after 6 years of age. This finding was not reported in patients with either OMOD1 or OMOD2. We consider that there may be a second independent disorder, but we have not found any pathogenic variants in the NF1 and NF2 genes in exome analysis.

In conclusion, the triad of (1) short upper limbs with dislocated radial head and limited elbow flexion/extension, (2) short first metacarpal bones, and (3) typical facial gestalt including a prominent forehead, short nose with a depressed broad nasal bridge with or without cleft lip and palate is suggestive for OMOD2. Additional features include short fibulae, short middle phalanges of the 5th fingers, and genitourinary anomalies. Our data support the notion that OMOD2 is a distinct clinical entity caused by mutations in the FZD2 gene.

\section{Statement of Ethics}

Written informed consent was obtained from the patient to participate in this study. This study was approved by the Charité Universitätsmedizin Berlin Ethics Committee.

\section{Disclosure Statement}

The authors have no conflicts of interest to disclose.
References

Autosomal Dominant Omodysplasia al Gazali LI, Abou al-Asaad F: Autosomal recessive omodysplasia. Clin Dysmorphol 4:52-56 (1995).

Berendsen AD, Olsen BR: Bone development. Bone 80:14-18 (2015).

Bonafe L, Cormier-Daire V, Hall C, Lachman R, Mortier G, et al: Nosology and classification of genetic skeletal disorders: 2015 revision. Am J Med Genet A 167A:2869-2892 (2015).

Campos-Xavier AB, Martinet D, Bateman J, Belluoccio D, Rowley L, et al: Mutations in the heparan-sulfate proteoglycan glypican 6 (GPC6) impair endochondral ossification and cause recessive omodysplasia. Am J Hum Genet 84:760-770 (2009).
Elçioglu NH, Gustavson KH, Wilkie AO, YükselApak M, Spranger JW: Recessive omodysplasia: five new cases and review of the literature. Pediatr Radiol 34:75-82 (2004).

Filmus J, Capurro M: The role of glypicans in Hedgehog signaling. Matrix Biol 35:248-252 (2014).

Geetha-Loganathan P, Nimmagadda S, Antoni L, Fu K, Whiting CJ, et al: Expression of WNT signalling pathway genes during chicken craniofacial development. Dev Dyn 238:11501165 (2009). 
Gordon BL, Champaigne NL, Rogers RC, Frias JL, Leroy JG: Long-term observation of a patient with dominant omodysplasia. Am J Med Genet A 164A:1234-1238 (2014).

Källberg M, Wang H, Wang S, Peng J, Wang Z, et al: Template-based protein structure modeling using the RaptorX web server. Nat Protoc 7:1511-1522 (2012).

Logan CY, Nusse R: The Wnt signaling pathway in development and disease. Annu Rev Cell Dev Biol 20:781-810 (2004).

Maroteaux P, Sauvegrain J, Chrispin A, Farriaux JP: Omodysplasia. Am J Med Genet 32:371375 (1989)

Person AD, Beiraghi S, Sieben CM, Hermanson S, Neumann AN, et al: WNT5A mutations in patients with autosomal dominant Robinow syndrome. Dev Dyn 239:327-337 (2010).

Robinson PN, Köhler S, Bauer S, Seelow D, Horn D, Mundlos S: The Human Phenotype Ontology: a tool for annotating and analyzing human hereditary disease. Am J Hum Genet 83: 610-615 (2008).
Roy A, Kucukural A, Zhang Y: I-TASSER: a unified platform for automated protein structure and function prediction. Nat Protoc 5:725738 (2010).

Saal HM, Prows CA, Guerreiro I, Donlin M, Knudson L, et al: A mutation in FRIZZLED2 impairs Wnt signaling and causes autosomal dominant omodysplasia. Hum Mol Genet 15: 24:3399-3409 (2015).

Sisson BE, Topczewski J: Expression of five frizzleds during zebrafish craniofacial development. Gene Expr Patterns 9:520-527 (2009).

Tauriello DV, Jordens I, Kirchner K, Slootstra JW, Kruitwagen T, et al: Wnt/ß-catenin signaling requires interaction of the Dishevelled DEP domain and C terminus with a discontinuous motif in Frizzled. Proc Natl Acad Sci USA 109:E812-820 (2012).

Venditti CP, Farmer J, Russell KL, Friedrich CA, Alter C, et al: Omodysplasia: an affected mother and son. Am J Med Genet 111:169177 (2002).
Yang J, Zhang Y: I-TASSER server: new development for protein structure and function predictions. Nucleic Acids Res 43:W174-181 (2015).

Yang J, Yan R, Roy A, Xu D, Poisson J, Zhang Y: The I-TASSER Suite: protein structure and function prediction. Nat Methods 12:7-8 (2015).

Yao T, Yang L, Li PQ, Wu H, Xie HB, et al: Association of Wnt3A gene variants with non-syndromic cleft lip with or without cleft palate in Chinese population. Arch Oral Biol 56:73-78 (2011).

Zemojtel T, Köhler S, Mackenroth L, Jäger M, Hecht J, et al: Effective diagnosis of genetic disease by computational phenotype analysis of the disease-associated genome. Sci Transl Med 6:252ra123 (2014).

Zhang Y: I-TASSER server for protein 3D structure prediction. BMC Bioinformatics 9:40 (2008). 